\title{
Intraspecific variation of gene structure in the mitochondrial large subunit ribosomal RNA and cytochrome c oxidase subunit 1 of Pyropia yezoensis (Bangiales, Rhodophyta)
}

\author{
Il Ki Hwang ${ }^{1}$, Seung-Oh Kim ${ }^{1}$, Mi Sook Hwang ${ }^{1}$, Eun-Jeong Park ${ }^{2}$, Dong-Soo Ha ${ }^{2}$ and \\ Sang-Rae Lee ${ }^{3, *}$ \\ ${ }^{1}$ Aquatic Plant Variety Center, National Institute of Fisheries Science, Mokpo 58746, Korea \\ ${ }^{2}$ Seaweed Research Center, National Institute of Fisheries Science, Mokpo 58746, Korea \\ ${ }^{3}$ Marine Research Institute, Pusan National University, Busan 46241, Korea
}

Red algal mitochondrial genomes (mtDNAs) can provide useful information on species identification. mtDNAs of Pyropia / Porphyra (Bangiales, Rhodophyta) have shown diverse variation in their size and gene structure. In particular, the introns and intronic open reading frames found in the ribosomal RNA large subunit gene $(r n l)$ and cytochrome $\mathrm{c}$ oxidase subunit 1 gene (coxl) significantly vary the mitochondrial genome size in Pyropia / Porphyra species. In this study, we examined the exon / intron structure of $r n l$ and coxl genes of Pyropia yezoensis at the intraspecific level. The combined data of $r n l$ and coxl genes exhibited 12 genotypes for 40 P. yezoensis strains, based on the existence of introns. These genotypes were more effective to identify P. yezoensis strains in comparison to the traditional DNA barcode cox 1 marker (5 haplotypes). Therefore, the variation in gene structure of $r n l$ and cox 1 can be a novel molecular marker to discriminate the strains of Pyropia species.

Key Words: coxl; intraspecific variation; intron; Pyropia yezoensis; rnl

\section{INTRODUCTION}

Pyropia species is one of the major seaweeds cultivated in Korea, Japan, and China (Niwa et al. 2004, Hwang et al. 2014). Strain identification at the intraspecific level of Pyropia yezoensis (Ueda) M. S. Hwang \& H. G. Choi is important to the Pyropia aquaculture industry for the maintenance of aquaculture strains and for the development of new cultivars. Although various molecular markers have been developed to discriminate P.yezoensis at the inter / intraspecific levels (Niwa et al. 2004, 2005, Hwang et al. 2005, Park et al. 2008, Niwa and Kobiyama 2009), more efficient tools for precise discrimination are still required.

Red algal mitochondrial genomes (mtDNAs) have interesting genes and structural composition (Odintsova and Yurina 2002, Smith et al. 2012, Yang et al. 2015). Such genetic features have provided useful information for interpreting the evolutionary history of Bangiophycean species (Smith et al. 2012). Particularly, the gene composition and structural variations of mtDNAs of Pyropia species have provided useful genetic information at the inter / intraspecific levels (Hwang et al. 2013, 2014, Hughey et al. 2014).
(9) $\$$ This is an Open Access article distributed under the terms of the Creative Commons Attribution Non-Commercial License (http://creativecommons.org/licenses/by-nc/3.0/) which permits unrestricted non-commercial use, distribution, and reproduction in any medium, provided the original work is properly cited.
Received October 29, 2017, Accepted February 20, 2018

* Corresponding Author

E-mail: oceanoalgae@naver.com

Tel: +82-51-510-3368, Fax: +82-51-581-2963 

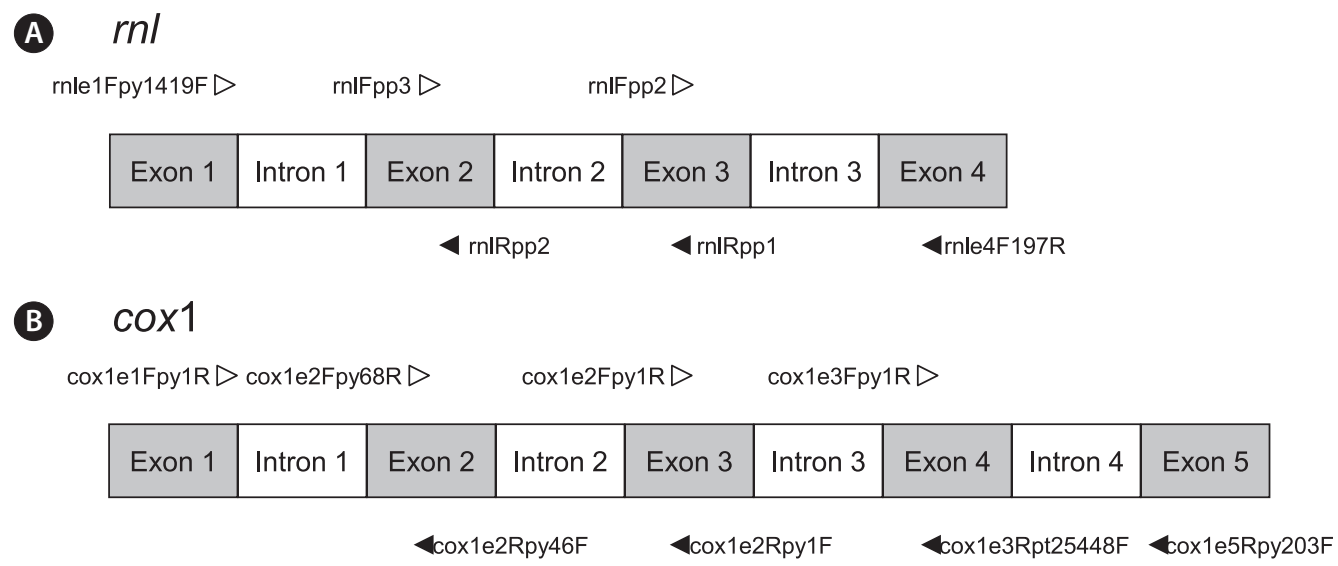

Fig. 1. Gene structure of $r n l(A)$ and $\operatorname{cox} 1$ (B) regions and the primer binding map. The exon / intron structures were re-analyzed using Hwang et al. $(2013,2014)$ as reference.

The study of mtDNAs in Pyropia / Porphyra (Bangiales, Rhodophyta) showed that they exhibit diverse variation in genome size and genetic structure, and also that they contain higher number of introns and intronic open reading frames (ORFs) in comparison to other red algae (Hughey et al. 2014, Hwang et al. 2014, Yang et al. 2015). Specifically, structural variation in the exon / intron structure of the large subunit ribosomal RNA gene $(r n l)$ and cytochrome c oxidase subunit 1 gene (cox 1 ) was reported in Pyropia / Porphyra species (Hwang et al. 2013, 2014, Hughey et al. 2014). These genetic variations significantly affected the size of mitochondrial genome of Pyropia / Porphyra species (Hwang et al. 2013, 2014, Hughey et al. 2014); a similar phenomenon was observed in the brown alga, Pylaiella littoralis (Ikuta et al. 2008).

Hwang et al. (2013, 2014) and Kong et al. (2014) examined the complete genome of mtDNA of $P$. tenera and $P$. yezoensis, and explored the genetic variation of exon / intron structure and the phylogenetic relationship among the intronic ORFs. Hughey et al. (2014) also reported the presence of intronic ORFs and the size variations of $\mathrm{mtD}$ NA of $P$. perforata. In this study, we examined the exon / intron structure of $r n l$ and coxl of P. yezoensis at the intraspecific level. The presence / absence of intron patterns were examined among the different strains of P.yezoensis.

\section{MATERIALS AND METHODS}

We analyzed 40 strains of $P$. yezoensis deposited in the Seaweed Research Center (National Institute of Fisheries Science, Mokpo, Korea) (Table 1). Blades of 27 P. yezoensis strains were collected from Korean coastal regions or aquaculture farms, and the conchocelis filaments were induced and cultured in the Provasoli enrichment medium (Provasoli 1968) at $20^{\circ} \mathrm{C}$ under white fluorescent irradiation of $20 \mu \mathrm{mol}$ photon $\mathrm{m}^{-2} \mathrm{~s}^{-1}$ (14 L : $10 \mathrm{D}$ cycle). Thirteen Japanese P. yezoensis strains were also analyzed (Table 1). The conchocelis filaments were used for the molecular analyses. Total genomic DNA was extracted using a DNeasy Plant Mini Kit (Qiagen, Hilden, Germany) following the manufacturer's instructions with an extension of incubation time by one hour.

To reveal the exon / intron structure of $r n l$ and cox 1 regions of $P$. yezoensis, we designed new primer sets having the binding sites on each exon region. These exonprimed intron-crossing (EPIC) markers showed high efficiency in revealing the exon / intron structure (Palumbi and Baker 1994, Bierne et al. 2000). Three and four primer sets were developed to reveal the genetic structure of $r n l$ and cox 1 regions, respectively (Tables $2 \& 3$, Fig. 1).

The mtDNAs of P. tenera (Kjellm.) N. Kikuchi, M. Miyata, M. S. Hwang \& H. G. Choi (NC_021475, Hwang et al. 2013) and P. yezoensis (NC_017837, Kong et al. 2014; KF561997, Hwang et al. 2014) were used as reference sequences for the primer design. We performed long range polymerase chain reaction (LPCR) to amplify the exon / intron structure of $r n l$ and coxl genes (Hwang et al. 2013). LPCR was carried out in a $20 \mu \mathrm{L}$ volume containing 10-50 ng of total genomic DNA, utilizing the LA Taq polymerase system (TaKaRa Bio, Shiga, Japan). The amplification conditions were as follows: $2 \mathrm{~min}$ at $94^{\circ} \mathrm{C}$, followed by 30 cycles at $94^{\circ} \mathrm{C}$ for $10 \mathrm{~s}, 60^{\circ} \mathrm{C}$ for $30 \mathrm{~s}$, and at $68^{\circ} \mathrm{C}$ for $5 \mathrm{~min}$, with a final extension at $68^{\circ} \mathrm{C}$ for $7 \mathrm{~min}$. Band patterns of LPCR products were analyzed by agarose gel electrophoresis. 
Table 1. Details of the samples analyzed in this study

\begin{tabular}{|c|c|c|c|c|c|c|}
\hline \multirow{2}{*}{ Species } & \multirow{2}{*}{$\begin{array}{l}\text { Strain } \\
\text { code }\end{array}$} & \multicolumn{3}{|c|}{ Genotype } & \multirow{2}{*}{ Sample site } & \multirow{2}{*}{ Sampling date } \\
\hline & & cox1 haplotype & $r n l$ and $c$ & 1 gene structure & & \\
\hline \multirow{27}{*}{$\begin{array}{l}\text { Korean Pyropia } \\
\text { yezoensis } \\
\text { (27 strains) }\end{array}$} & GB-1 & $\mathrm{C} 1$ & H1 & R011C0100 & Songdo, Pohang, Gyeongbuk & Feb 6, 2001 \\
\hline & GB-2 & $\mathrm{C} 2$ & $\mathrm{H} 2^{\mathrm{a}}$ & R101C1101 & Masanri, Pohang, Gyeongbuk & Apr 3, 2008 \\
\hline & GN-1 & C3 & H1 & R011C0100 & Neungpo, Geoje, Gyeongnam & Feb 5, 2001 \\
\hline & GN-2 & $\mathrm{C} 1$ & H3 & R010C0100 & Galmok, Tongyeong, Gyeongnam & Feb 7, 2001 \\
\hline & GN-3 & $\mathrm{C} 1$ & H3 & R010C0100 & Sachon, Namhae, Gyeongnam & Feb 29, 2004 \\
\hline & JN-1 & C3 & $\mathrm{H} 4$ & R110C0001 & Cheongsando, Wando, Jeonnam & Feb 19, 2004 \\
\hline & $\mathrm{JN}-2$ & C3 & H5 & R111C0000 & Maenggoldo, Jindo, Jeonnam & Apr 1, 2007 \\
\hline & $\mathrm{JN}-3$ & C3 & H6 & R101C0101 & Hajodo, Jindo, Jeonnam & Mar 19, 2004 \\
\hline & $\mathrm{JN}-4$ & C3 & $\mathrm{H} 7$ & R111C0001 & Euisin, Jindo, Jeonnam & Feb 26, 2000 \\
\hline & $\mathrm{JN}-5$ & C3 & H5 & R111C0000 & Songji-1, Haenam, Jeonnam & Feb 25, 2011 \\
\hline & JN-6 & C3 & H5 & R111C0000 & Songji-2, Haenam, Jeonnam & Mar 15, 2012 \\
\hline & $\mathrm{JN}-7$ & C3 & H5 & R111C0000 & Songji-3, Haenam, Jeonnam & Feb 18, 2008 \\
\hline & $\mathrm{JN}-8$ & C3 & H8 & R111C0101 & Songji-4, Haenam, Jeonnam & Feb 20, 2009 \\
\hline & JN-9 & C3 & H5 & R111C0000 & Songji-5, Haenam, Jeonnam & Feb 22, 2008 \\
\hline & $\mathrm{JN}-10$ & C3 & H5 & R111C0000 & Imhado-1, Haenam, Jeonnam & Mar 10, 2014 \\
\hline & JN-11 & C3 & H5 & R111C0000 & Imhado-2, Haenam, Jeonnam & Mar 10, 2014 \\
\hline & JN-12 & C3 & H9 & R111C0100 & Hwawon, Haenam, Jeonnam & Mar 12, 2002 \\
\hline & JN-13 & C3 & H5 & R111C0000 & Madong-1, Muan, Jeonnam & Feb 27, 2013 \\
\hline & JN-14 & C3 & H5 & R111C0000 & Madong-2, Muan, Jeonnam & Feb 28, 2012 \\
\hline & JN-15 & C3 & $\mathrm{H} 12$ & R101C0111 & Dochodo, Sinan, Jeonnam & Mar 4, 2011 \\
\hline & JN-16 & C3 & H12 & R101C0111 & Bigeumdo, Sinan, Jeonnam & Mar 5, 2011 \\
\hline & $\mathrm{JN}-17$ & $\mathrm{C} 1$ & H12 & R101C0111 & Heuksando, Sinan, Jeonnam & Jan 30, 2009 \\
\hline & JB-1 & C3 & $\mathrm{H} 10$ & R101C0100 & Wido-1, Buan, Jeonbuk & Apr 7, 2004 \\
\hline & JB-2 & C3 & $\mathrm{H} 10$ & R101C0100 & Wido-2, Buan, Jeonbuk & Apr 7, 2004 \\
\hline & JB-3 & C3 & H8 & R111C0101 & Munyeodo, Gunsan, Jeonbuk & Apr 8, 2004 \\
\hline & $\mathrm{CN}-1$ & $\mathrm{C} 4$ & H11 & R110C0101 & Daecheon-1, Chungnam & Apr 22, 2004 \\
\hline & $\mathrm{CN}-2$ & $\mathrm{C} 4$ & H11 & R110C0101 & Daecheon-2, Chungnam & Apr 22, 2004 \\
\hline \multirow{13}{*}{$\begin{array}{l}\text { Japanese } \\
\text { P. yezoensis } \\
\text { (13 strains) }\end{array}$} & JP-Tul & C5 & $\mathrm{H} 12$ & R101C0111 & Tu-1, Kisarazu, Chiba, Japan & $\operatorname{Mar} 9,1974^{b}$ \\
\hline & JP-PYN & C5 & H12 & R101C0111 & $\begin{array}{l}\text { Aquaculture strain of P. yezoensis } \\
\text { f. narawaensis from Japan }\end{array}$ & $2000^{c}$ \\
\hline & JP-PY1 & C5 & H12 & R101C0111 & Same as above & $2004^{\mathrm{c}}$ \\
\hline & JP-PY2 & C5 & H12 & R101C0111 & Same as above & $2004^{\mathrm{c}}$ \\
\hline & JP-PY3 & C5 & $\mathrm{H} 12$ & R101C0111 & Same as above & $2004^{c}$ \\
\hline & JP-PY4 & C5 & $\mathrm{H} 12$ & R101C0111 & Same as above & $2008^{\mathrm{c}}$ \\
\hline & JP-PY5 & C5 & $\mathrm{H} 12$ & R101C0111 & Same as above & $2008^{\mathrm{c}}$ \\
\hline & JP-PY6 & C5 & $\mathrm{H} 12$ & R101C0111 & Same as above & $2007^{\mathrm{c}}$ \\
\hline & JP-PY7 & C5 & $\mathrm{H} 12$ & R101C0111 & Same as above & $2007^{\mathrm{c}}$ \\
\hline & JP-PY8 & C5 & H12 & R101C0111 & Same as above & $2007^{c}$ \\
\hline & JP-PY9 & C5 & H12 & R101C0111 & Same as above & $2007^{\mathrm{c}}$ \\
\hline & JP-PY10 & C5 & H12 & R101C0111 & Same as above & $2007^{\mathrm{c}}$ \\
\hline & JP-PY11 & C5 & H12 & R101C0111 & Same as above & $2007^{\mathrm{c}}$ \\
\hline
\end{tabular}

The structural variation of the gene is represented as a code $(\mathrm{R}=r n l, C=\operatorname{cox} 1,0=$ intron absent, $1=$ intron present).

GenBank accession numbers for five types of cox1 sequences (C1-C5, MF663741-MF663745).

Electrophoresis gel images were represented in the Supplementary Fig. S1.

${ }^{\mathrm{a}} \mathrm{H} 2$ genotype showed the different length in introns.

${ }^{\mathrm{b}}$ This sample was obtained from Prof. Saga in Hokkaido University, Japan.

'These were obtained from a company that cultures free living conchocelis filaments of Pyropia.

Table 2. Details of the primers used for the amplification of $r n l$ and cox 1 regions

\begin{tabular}{lllll}
\hline Gene region $^{\text {a }}$ & Forward primer & \multicolumn{1}{c}{ Sequence $\left(\mathbf{5}^{\prime}\right.$ to $\left.\mathbf{3}^{\prime}\right)$} & Reverse primer & \multicolumn{1}{c}{ Sequence $\left(5^{\prime}\right.$ to $\left.\mathbf{3}^{\prime}\right)$} \\
\hline$r n l(\mathrm{e} 1)-(\mathrm{e} 2)$ & rnle1Fpyl419F & ACTCGGCAAATTTACTCCGTAC & rnlRpp2 & CATGATAAATCTGTTATCCCTAGAG \\
$r n l(\mathrm{e} 2)-(\mathrm{e} 3)$ & rnlFpp3 & CCTCCTAAAGTGTAACGGAGGTG & rnlRpp1 & ACTGTCTCACGACGTTCTGAACC \\
$r n l(\mathrm{e} 3)-(\mathrm{e} 4)$ & rnlFpp2 & GAACGTCGTGAGACAGTTCGGTC & rnle4F197R & GCAAGAAACAATACAACCGATACAC \\
$\operatorname{cox} 1(\mathrm{e} 1)-(\mathrm{e} 2)$ & coxle1Fpy1R & CTCGACCAATCATAAAGATATAGG & coxle2Rpy46F & AATACAGGATCGCCACCACC \\
$\operatorname{cox} 1(\mathrm{e} 2)-(\mathrm{e} 3)$ & coxle2Fpy68R & TCAGGTGGTGGCGATCCTGT & coxle2Rpy1F & ACCTATAGAAAGCATAGCATAAATC \\
$\operatorname{cox} 1(\mathrm{e} 3)-(\mathrm{e} 4)$ & coxle2Fpy1R & ATGATTTATGCTATGCTTTCTATAGG & coxle3Rpt25448F & AGCTAGTATAATACCAGTAAGTCC \\
$\operatorname{cox} 1(\mathrm{e} 4)-(\mathrm{e} 5)$ & coxle3Fpy1R & TGGATTGCTACTATGTGAGAAGG & coxle5Rpy203F & CTAAATAACGCAACATATGAACC \\
\hline
\end{tabular}

${ }^{a}$ We followed the results of Hwang et al. (2014) for nomenclature to present the structural variation. 
We also determined the sequences of the cox 1 in 40 $P$. yezoensis strains, which has been used as the standard target DNA region for DNA barcoding of red algae (Saunders 2005). To amplify the cox 1 region, we designed a new primer, coxlelR16F (5'-TGCCAAGACAGGTACTGCT-3') having the binding position from 30738 to 30756 in P. yezoensis (NC_017837), located at the 5' end of cox 1 gene. The primer pair, coxle1R16F (this study) and coxle1FpylR (Hwang et al. 2013), was used for the amplification and sequencing of coxl region in P. yezoensis samples. The amplification conditions were as follows: 3 min at $95^{\circ} \mathrm{C}$, followed by 40 cycles at $94^{\circ} \mathrm{C}$ for $30 \mathrm{~s}, 50^{\circ} \mathrm{C}$ for $30 \mathrm{~s}$, and $72^{\circ} \mathrm{C}$ for $1 \mathrm{~min}$, with a final extension at $72^{\circ} \mathrm{C}$ for $7 \mathrm{~min}$. PCR products were sequenced commerically (Genotech, Daejeon, Korea) and the sequences were assembled in Sequencher 5.4.6 (Gene Codes Corporation, Ann Arbor, MI, USA). Pairwise distances were calculated using the MEGA 6.0 program (Tamura et al. 2013).

\section{RESULTS}

PCR amplification of coxl gene generated a $549 \mathrm{bp}$ in all 40 samples, excluding the primer binding sites. Analysis of coxl sequences revealed 4 haplotypes among the 27 Korean P. yezoensis strains (C1-C4) (Table 1). On the other hand, 13 strains of Japanese $P$. yezoensis shared the same haplotype (C5). The pairwise distance among the haplotypes ranged from 0.2 to $0.9 \%$ (1-5 bp).

We found four exons and three introns in the $r n l$ in all of the sampled P. yezoensis strains, also reported in previous studies (Hwang et al. 2013, 2014); and five exons and four introns were revealed in the $\operatorname{cox} 1$ (Fig. 1). The results revealed five genotypes (R111, R110, R101, R011, and R010) in the $r n l$ intron and six genotypes (C1101, C0111, C0101, C0100, C0001, and C0000) in the cox intron based on the existence of introns (Tables 1 \& 3). Combined structural variations of $r n l$ and coxl exhibited 12 genotypes among the 40 P. yezoensis strains (Table 1). Korean P. yezoensis exhibited 12 genotypes (H1-H12) among 27 strains. The genotype 5 (H5) was dominant, and broadly distributed in nine Korean strains. All of the Japanese P. yezoensis strains had the same gene structure (H12) (Table 1).

\section{DISCUSSION}

The presence of introns and intronic ORFs is the main reason for variation in the size of the mitochondrial genome of Pyropia species. Hwang et al. (2013, 2014) was the first to report this intron structure from P. tenera and $P$. yezoensis in red algae. In this study, we examined the genetic features of intron structure of $r n l$ and $\operatorname{cox} 1$ of $P$. yezoensis at the intraspecific level, and found 12 genetic types from 40 culture strains from Korea and Japan (Tables 1 \& 3).

The sequences of mitochondrial coxl region have been recommended as DNA barcode markers to identify animal and algal species, and the coxl region exhibited high efficiency in describing species boundaries (Hebert et al. 2003, Saunders 2005). In this study, five haplotypes of the coxl gene were revealed for 40 P. yezoensis strains (Table 1). Korean P. yezoensis exhibited 4 haplotypes

Table 3. Primer position and the predicted amplicon size

\begin{tabular}{|c|c|c|c|c|c|c|}
\hline \multirow{2}{*}{ Gene region } & \multicolumn{4}{|c|}{ PCR primer position $^{a}$} & \multicolumn{2}{|c|}{ Amplicon size (bp) } \\
\hline & Primer & Binding site & Primer & Binding site & Intron (present) & Intron (absent) \\
\hline$r n l(\mathrm{e} 1)-(\mathrm{e} 2)$ & rnle1Fpy1419F & $1,419-1,440$ & rnlRpp2 & 4,441-4,465 & 3,047 & 720 \\
\hline$r n l(\mathrm{e} 2)-(\mathrm{e} 3)$ & rnlFpp3 & $4,260-4,282$ & rnlRpp1 & $4,583-4,605$ & $2,842^{\mathrm{b}}$ & 346 \\
\hline$r n l(\mathrm{e} 3)-(\mathrm{e} 4)$ & rnlFpp2 & $4,589-4,611$ & rnle4F197R & 7,174-7,198 & 2,610 & 120 \\
\hline $\operatorname{cox} 1(\mathrm{e} 1)-(\mathrm{e} 2)$ & coxlelFpylR & $31,306-31,329$ & coxle2Rpy46F & $30,658-30,677$ & $3,258^{\mathrm{c}}$ & 672 \\
\hline $\operatorname{cox} 1(\mathrm{e} 2)-(\mathrm{e} 3)$ & coxle2Fpy68R & $30,661-30,680$ & coxle2Rpy1F & $27,998-28,022$ & 2,664 & 174 \\
\hline $\operatorname{cox} 1(\mathrm{e} 3)-(\mathrm{e} 4)$ & coxle2Fpy1R & $27,999-28,024$ & coxle3Rpt25448F & $25,448-25,471$ & 2,577 & 261 \\
\hline $\operatorname{cox} 1(\mathrm{e} 4)-(\mathrm{e} 5)$ & coxle3Fpy1R & $25,536-25,558$ & coxle5Rpy203F & $22,773-22,795$ & 2,786 & 425 \\
\hline
\end{tabular}

$\mathrm{PCR}$, polymerase chain reaction.

${ }^{a}$ The primer position and the predicted amplicon size was determined using the mitochondrial genome (mtDNA) of Pyropia yezoensis (NC_017837) as a reference.

${ }^{\mathrm{b}}$ Pyropia yezoensis (KF561997, Hwang et al. 2014) was used as a reference, because P. yezoensis (NC_017837) does not have an intron between exon 2 (e2) and exon 3 (e3) of $r n l$ gene (Hwang et al. 2014).

'Pyropia tenera (NC_021475) was used as a reference. Pyropia yezoensis (NC_01783, KF561997) does not have an intron between exon 1 (e1) and exon 2 (e2) of cox1 gene (Hwang et al. 2014). 
(haplotypes C1-C4) for 27 strains with $0.2-0.9 \%$ pairwise genetic distances. All the 13 Japanese P. yezoensis strains had the same cox 1 sequence (haplotype C5), and showed $0.2-0.7 \%$ pairwise distances from the Korean haplotypes.

Some of the P. yezoensis strains yielded the same cox 1 sequence, but different introns (Table 1). The most variable haplotype $\mathrm{C} 3$ of cox 1 was sub-divided into nine genotypes (H1, H4, H5, H6, H7, H8, H9, H10, and H12), using the combined $r n l$ and coxl. The genotype $\mathrm{Cl}$ was subdivided into three genotypes ( $\mathrm{H} 1, \mathrm{H} 3$, and $\mathrm{H} 12)$. Therefore, the gene structure of the $r n l$ and coxl regions exhibited higher genetic resolution to discriminate $P$. yezoensis strains in comparison to the cox 1 sequence variations in these samples.

Besides cox 1 sequences, several genetic markers discriminating the Pyropia / Porphyra strains at the inter / intra-specific level, such as SSU ribosomal DNA (rDNA), ITS1, $r b c \mathrm{~L}$, and the RuBisCO spacer have been studied (Brodie et al. 1998, Lindstrom and Fredericq 2003, Hwang et al. 2005, Nelson et al. 2006, Niwa et al. 2009, Sutherland et al. 2011, Kucera and Saunders 2012, Mols-Mortensen et al. 2014, Guillemin et al. 2016). Nuclear SSU rDNA and $r b c \mathrm{~L}$ sequences were shown to discriminate the Korean $P$. yezoensis strains from the Japanese $P$. yezoensis strains (Hwang et al. 2005). In this study, coxl sequence analysis divided the 27 Korean P. yezoensis strains into four types; the presence / absence of introns in $r n l$ and coxl genes divided the 27 Korean P. yezoensis strains into 12 types; and the combination of both markers divided the Korean $P$. yezoensis strains into 14 types. These results implied that the combination of both markers was more useful in comparison to a single marker.

On the other hand, only one genotype was found in all the Japanese P. yezoensis strains, which was in contrast to the Korean strains. All Japanese P. yezoensis strains were aquaculture strains, except JP-Tul. Niwa et al. (2008, 2009) reported the presence of three genotypes in 13 Japanese aquaculture strains of P. yezoensis; 11 strains had the same type, and each of the remaining two strains had a unique type based on ITS1 region analysis. The Japanese materials used in this study had only one genotype, which is the same type as that of P. yezoensis f. narawaensis reported by Niwa et al. (2008, 2009).

Theoretically, the presence / absence of introns can produce eight types in the $r n l$ and 16 types in the coxl, and 128 types from the combination of $r n l$ and $\operatorname{cox} 1$ of $P$. yezoensis. Additional genotypes of intron could be found across global samplings of $P$. yezoensis strains, including other Pyropia species in Korea, Japan, and China. In this study we found high intraspecific genetic variation in the $r n l$ and coxl genes, and showed that these two genes can better discriminate strains of $P$. yezoensis. These molecular markers can be useful to maintain the diversity of aquaculture strains and for the development of new cultivars.

\section{ACKNOWLEDGEMENTS}

This work was supported by a grant from the National Institute of Fisheries Science (R2018011 \& P2018011), Korea.

\section{SUPPLEMENTARY MATERIAL}

Supplementary Fig. S1. Electrophoresis gel images of the genotypes of $r n l$ and coxl from Pyropia yezoensis strains. Two PCR markers were also loaded in the gel (GeneRuler 1 kb DNA ladder [left; ThermoFisher Scientific, USA] and GeneRuler 100 bp Plus DNA ladder [middle]) (http://www.e-algae.org).

\section{REFERENCES}

Bierne, N., Lehnert, S. A., Bédier, E., Bonhomme, F. \& Moore, S. S. 2000. Screening for intron-length polymorphisms in penaeid shrimps using exon-primed intron-crossing (EPIC)-PCR. Mol. Ecol. 9:233-235.

Brodie, J., Hayes, P. K., Barker, G. L., Irvine, L. M. \& Bartsch, I. 1998. A reappraisal of Porphyra and Bangia (Bangiophycidae, Rhodophyta) in the Northeast Atlantic based on the $r b c \mathrm{~L}-r b c S$ intergenic spacer. J. Phycol. 34:1069-1074.

Guillemin, M. L., Contreras-Porcia, L., Ramírez, M. E., Macaya, E. C., Contador, C. B., Woods, H., Wyatt, C. \& Brodie, J. 2016. The bladed Bangiales (Rhodophyta) of the South Eastern Pacific: molecular species delimitation reveals extensive diversity. Mol. Phylogenet. Evol. 94:814-826.

Hebert, P. D. N., Cywinska, A., Ball, S. L. \& deWaard, J. R. 2003. Biological identifications through DNA barcodes. Proc. Biol. Sci. 270:313-321.

Hughey, J. R., Gabrielson, P. W., Rohmer, L., Tortolani, J., Silva, M., Miller, K. A., Young, J. D., Martell, C. \& Ruediger, E. 2014. Minimally destructive sampling of type specimens of Pyropia (Bangiales, Rhodophyta) recovers complete plastid and mitochondrial genomes. Sci. Rep. 4:5113.

Hwang, M. S., Kim, S. -M., Ha, D. -S., Baek, J. M., Kim, H. -S. 
\& Choi, H. -G. 2005. DNA sequences and identification of Porphyra cultivated by natural seeding on the southwest coast of Korea. Algae 20:183-196.

Hwang, M. S., Kim, S. -O., Ha, D. -S., Lee, J. E. \& Lee, S. -R. 2013. Complete sequence and genetic features of the mitochondrial genome of Pyropia tenera (Rhodophyta). Plant Biotechnol. Rep. 7:435-443.

Hwang, M. S., Kim, S. -O., Ha, D. -S., Lee, J. E. \& Lee, S. -R. 2014. Complete mitochondrial genome sequence of Pyropia yezoensis (Bangiales, Rhodophyta) from Korea. Plant Biotechnol. Rep. 8:221-227.

Ikuta, K., Kawai, H., Müller, D. G. \& Ohama, T. 2008. Recurrent invasion of mitochondrial group II introns in specimens of Pylaiella littoralis (brown alga), collected worldwide. Curr. Genet. 53:207-216.

Kong, F., Sun, P., Cao, M., Wang, L. \& Mao, Y. 2014. Complete mitochondrial genome of Pyropia yezoensis: reasserting the revision of genus Porphyra. Mitochondrial DNA 25:335-336.

Kucera, H. \& Saunders, G. W. 2012. A survey of Bangiales (Rhodophyta) based on multiple molecular markers reveals cryptic diversity. J. Phycol. 48:869-882.

Lindstrom, S. C. \& Fredericq, S. 2003. rbcL gene sequence reveal relationships among north-east Pacific species of Porphyra (Bangiales, Rhodophyta) and a new species, $P$. aestivalis. Phycol. Res. 51:211-224.

Mols-Mortensen, A., Neefus, C. D., Pedersen, P. M. \& Brodie, J. 2014. Diversity and distribution of foliose Bangiales (Rhodophyta) in West Greenland: a link between the North Atlantic and North Pacific. Eur. J. Phycol. 49:1-10.

Nelson, W. A., Farr, T. J. \& Broom, J. E. S. 2006. Phylogenetic relationships and generic concepts in the red order Bangiales: challenges ahead. Phycologia 45:249-259.

Niwa, K., Iida, S., Kato, A., Kawai, H., Kikuchi, N., Kobiyama, A. \& Aruga, Y. 2009. Genetic diversity and introgression in two cultivated species (Porphyra yezoensis and Porphyra tenera) and closely related wild species of Porphyra (Bangiales, Rhodophyta). J. Phycol. 45:493-502.

Niwa, K., Kato, A., Kobiyama, A., Kawai, H. \& Aruga, Y. 2008. Comparative study of wild and cultivated Porphyra yezoensis (Bangiales, Rhodophyta) based on molecular and morphological data. J. Appl. Phycol. 20:261-270.

Niwa, K., Kikuchi, N., Iwabuchi, M. \& Aruga, Y. 2004. Morphological and AFLP variation of Porphyra yezoensis Ueda form, narawaensis Miura (Bangiales, Rhodophyta). Phycol. Res. 52:180-190.

Niwa, K. \& Kobiyama, A. 2009. Simple molecular discrimina- tion of cultivated Porphyra species (Porphyra yezoensis and Porphyra tenera) and related wild species (Bangiales, Rhodophyta). Phycol. Res. 57:299-303.

Niwa, K., Kobiyama, A. \& Aruga, Y. 2005. Confirmation of cultivated Porphyra tenera (Bangiales, Rhodophyta) by polymerase chain reaction restriction fragment length polymorphism analyses of the plastid and nuclear DNA. Phycolo. Res. 53:296-302.

Odintsova, M. S. \& Yurina, N. P. 2002. The mitochondrial genome of protists. Russ. J. Genet. 38:642-655.

Palumbi, S. R. \& Baker, C. S. 1994. Contrasting population structure from nuclear intron sequences and mtDNA of humpback whales. Mol. Biol. Evol. 11:426-435.

Park, E. -J., Endo, H., Kitade, Y. \& Saga, N. 2008. Simple differentiation of two closely related species Porphyra tenera and Porphyra yezoensis (Bangiophyceae, Rhodophyta) based on length polymorphism of actin-related protein 4 gene (ARP4). Fish. Sci. 74:613-620.

Provasoli, L. 1968. Media and prospects for the cultivation of marine algae. In Watanabe, A. \& Hattori, A. (Eds.) Culture and Collections of Algae. Proc. U.S.-Japan Conference, Japanese Society of Plant Physiology, Hakone, pp. 63-75.

Saunders, G. W. 2005. Applying DNA barcoding to red macroalgae: a preliminary appraisal holds promise for future applications. Philos. Trans. R. Soc. Lond. B Biol. Sci. 360:1879-1888.

Smith, D. R., Hua, J., Lee, R. W. \& Keeling, P. J. 2012. Relative rates of evolution among the three genetic compartments of the red alga Porphyra differ from those of green plants and do not correlate with genome architecture. Mol. Phylogenet. Evol. 65:339-344.

Sutherland, J. E., Lindstrom, S. C., Nelson, W. A., Brodie, J., Lynch, M. D. J., Hwang, M. S., Choi, H. -G., Miyata, M., Kikuchi, N., Oliveira, M. C., Farr, T., Neefus, C., MolsMortensen, A., Milstein, D. \& Müller, K. M. 2011. A new look at an ancient order: generic revision of the Bangiales (Rhodophyta). J. Phycol. 47:1131-1151.

Tamura, K., Stecher, G., Peterson, D., Filipski, A. \& Kumar, S. 2013. MEGA6: Molecular Evolutionary Genetics Analysis version 6.0. Mol. Biol. Evol. 30:2725-2729.

Yang, E. C., Kim, K. M., Kim, S. Y., Lee, J., Boo, G. H., Lee, J. -H., Nelson, W. A., Yi, G., Schmidt, W. E., Fredericq, S., Boo, S. M., Bhattacharya, D. \& Yoon, H. S. 2015. Highly conserved mitochondrial genomes among multicellular red algae of the Florideophyceae. Genome Biol. Evol. 7:2394-2406. 